\title{
Die Arztpraxis in der Rechtsform einer Aktiengesellschaft
}

\author{
Gesundheitsrechtliche, zivilrechtliche und steuerrechtliche Aspekte
}

B. Rieder ${ }^{a}$, U. Eggenberger Stöcklib , G. De Paolis ${ }^{c}$

\section{Einleitung}

Eine Reihe von kürzlich in Kraft getretenen Gesetzesänderungen machen es für Ärzte rechtlich möglich und allenfalls sinnvoll, ihre Praxis, die sie bisher in der Rechtsform einer Personengesellschaft (Einzelfirma, einfache Gesellschaft oder Kollektivgesellschaft) geführt haben, neu in der Rechtsform einer juristischen Person (AG oder $\mathrm{GmbH}$ ) weiterzuführen. Die damit verbundenen gesundheitsrechtlichen, haftungsrechtlichen und insbesondere steuerrechtlichen Aspekte sollen nachfolgend kurz beleuchtet werden.

\section{Gesundheitsrechtliche Aspekte}

\section{Krankenversicherungsgesetz}

Nach dem Krankenversicherungsgesetz (KVG) können Ärzte (und Ärztinnen, welche im folgenden mitgemeint sind) seit dem 1. Januar 2001 auch ausserhalb eines Spitals in Anstellungsverhältnissen tätig sein. Möglich macht dies eine Ergänzung, welche Einrichtungen, die der ambulanten Krankenpflege durch Ärzte dienen, als Leistungserbringer in der Grundversorgung zulässt. Voraussetzung ist, dass die dort tätigen Ärzte über das eidgenössische Diplom oder einen gleichwertigen wissenschaftlichen Befähigungsausweis sowie eine anerkannte Weiterbildung verfügen. Nebst diesen Grundvoraussetzungen ist der neue Artikel 55a des KVG («Zulassungsstopp») zu beachten. Dieser gibt die Möglichkeit, die Zulassung von Leistungserbringern einzuschränken. Für Einrichtungen, die der ambulanten Krankenpflege dienen, gilt diese einschränkende Regelung ebenfalls.

Anlass für diese Ergänzung gaben Entwicklungen bei den besonderen Versicherungsformen und ihren vielfältigen Institutionen. Das KVG setzte die selbständige (Erwerbs-)Tätigkeit von Ärzten zwar nie zwingend voraus, bisher fehlte jedoch eine gesetzliche Grundlage, um Ärzte in einem vertraglichen Anstellungsverhältnis ausserhalb eines Spitals oder einer ande- ren Einrichtung der stationären oder teilstationären Krankenpflege als Leistungserbringer anzuerkennen. Diese Grundlage wurde mit den neuen Artikeln im KVG nun geschaffen. Damit besteht vom Gesetz her die Möglichkeit, auch als angestellter Arzt, beispielsweise in einer Ärzteaktiengesellschaft, Leistungen unter dem KVG zu erbringen und über die obligatorische Grundversicherung abzurechnen. Der Rahmenvertrag TARMED ermöglicht die Zusammenarbeit von Ärzten in Form einer juristischen Person ebenfalls.

\section{Kantonale Vorschriften}

Das Krankenversicherungsgesetz als Bundesgesetz gilt zwar in der ganzen Schweiz, die Bewilligungen für die Berufsausübung und die Praxisführung unterstehen jedoch weiterhin dem kantonalen Recht. Und hier sind nun Unterschiede auszumachen. Im Kanton Zürich beispielsweise verlangen sowohl Gesundheitsgesetz als auch die Ärzteverordnung, dass die ärztliche Tätigkeit als selbständige Tätigkeit ausgeübt wird. Das bernische Recht demgegenüber verlangt nur die eigene fachliche Verantwortung, nicht hingegen, dass die ärztliche Tätigkeit in selbständiger Stellung ausgeübt wird; eine Anstellung von Ärzten und die Zusammenarbeit in der Form einer juristischen Person sind hier also möglich. Anfragen an verschiedene andere Kantone haben ergeben, dass teilweise die Rechtslage in den Kantonen bezüglich der Zulässigkeit von juristischen Personen als Leistungserbringer einer ambulanten Krankenpflege noch völlig unklar ist bzw. die entsprechenden kantonalen Gesetzesnormen noch nicht an die neuen Regelungen des KVG angepasst wurden. Es bleibt zu hoffen, dass derartige kantonale Unterschiede in $\mathrm{Zu}$ kunft weiter abgebaut werden.

\section{Praktisches Vorgehen}

Was ist nun praktisch vorzukehren, damit die im Rahmen einer unselbständigen Erwerbstätigkeit erbrachten Leistungen über die Grundversicherung abgerechnet werden können? 
Als erstes sollte geprüft werden, ob das anwendbare kantonale Recht die Zusammenarbeit in der Form einer juristischen Person zulässt. Wie vorstehend aufgezeigt, kann es Unterschiede geben.

Ist diese Form der Tätigkeit zulässig, ist weiter abzuklären, welche Bewilligungen erforderlich sind. Teilweise reicht die persönliche Berufsausübungsbewilligung der einzelnen Ärzte aus, teilweise sind Praxisbewilligungen erforderlich oder es muss - bei Neugründung einer Praxis das Domizil angezeigt werden. In jedem Fall benötigt die juristische Person eine Registernummer von santésuisse. Diese erhält sie unter Angabe der bisherigen Registernummern der beteiligten Ärzte sowie eines Handelsregisterauszugs bei der zuständigen Stelle von santésuisse. Als letzter Schritt folgt dann der Beitritt zum TARMED bzw. dem entsprechenden kantonalen Anschlussvertrag. Mit diesen Vorkehrungen kann die juristische Person schliesslich unter Angabe ihrer Registernummer sowie der persönlichen EAN-Nummer des behandelnden Arztes Rechnung stellen.

\section{Zivilrechtliche, insbesondere haftungs- rechtliche Aspekte}

Die nachfolgenden Ausführungen beziehen sich grundsätzlich auf das Rechtskleid einer Aktiengesellschaft (AG). Sie gelten sinngemäss auch für die Rechtsform der Gesellschaft mit beschränkter Haftung. Die AG ist eine sogenannt juristische Person, was bedeutet, dass die Gesellschaft Trägerin aller Rechte und Pflichten ist und für deren Verbindlichkeiten nur das Gesellschaftsvermögen haftet. Das Mindestkapital einer AG beträgt Fr. 100000.- Nennwert. Davon müssen mindestens Fr. 50000.- einbezahlt werden.

Die Organe der Gesellschaft sind die Generalversammlung (oberstes Organ = Versammlung aller Aktionäre), der Verwaltungsrat (geschäftsführendes Organ = Aktionäre, welche die AG effektiv leiten) und die Revisionsstelle (qualifizierte und unabhängige Dritte, welche die Buchführung, Jahresrechnung und Gewinnverwendung prüfen).

Die AG wird errichtet, indem mindestens drei Gründer in öffentlicher Urkunde erklären, eine Aktiengesellschaft zu gründen, darin die Statuten festlegen und die Organe bestellen. Die Gesellschaft erlangt das Recht der Persönlichkeit durch die Eintragung in das Handelsregister.

Das Aktienkapital der Gesellschaft kann mittels Bareinlage oder Sacheinlage liberiert werden. Die Gründung mittels Sacheinlage (Einbringung von mobilen Sachanlagen, Forderungen, Finanzanlagen, Grundstücken, Immaterialgüterrechten usw.) ist typischerweise bei der Umwandlung einer Einzelfirma (Arztpraxis) in eine Aktiengesellschaft der Fall, so dass für die Gründung einer Aktiengesellschaft keine zusätzlichen Barmittel erforderlich sind.

Für die Aktiengesellschaft gelten spezielle Rechnungslegungsvorschriften. Die Gesellschaft hat jährlich einen Geschäftsbericht zu erstellen, welcher sich aus der Jahresrechnung (Bilanz, Erfolgsrechnung, Anhang) und einem Jahresbericht zusammensetzt.

Die beteiligten Aktionäre können untereinander Verpflichtungen eingehen, um die Stimmrechte oder die Verfügungsrechte über die Aktien, die Vertretung im Verwaltungsrat, die Geschäftspolitik in gewünschte Bahnen zu lenken und sicherzustellen. Ein sogenannter Aktionärsbindungsvertrag eignet sich daher gut, um bisherige im Gesellschaftervertrag geregelte Rechte und Pflichten weiterhin bindend zwischen den Aktionären zu vereinbaren.

Mit Bezug auf haftungsrechtliche Aspekte lässt sich festhalten, dass die bisher bekannten Ausgestaltungen der vertraglichen Haftung sich entweder als Haftung zwischen dem Arzt, der seine Praxis in der Rechtsform einer Personengesellschaft führt, und seinem Patienten oder zwischen der Klinik als Arbeitgeberin des Arztes und dem Patienten gestalten. Zwischen dem angestellten Arzt und dem Klinikpatienten kommt in der Regel kein Vertragsverhältnis zustande. Im Schadenfall wird der geschädigte Patient in erster Linie seinen Vertragspartner, also entweder den selbständigen Arzt oder die Klinik, ins Recht fassen.

In beiden Fällen kommt zwischen den Vertragsparteien, sei es Arzt - Patient oder Klinik Patient, regelmässig ein Vertrag nach Auftragsverhältnis zustande. Hiernach haftet der Beauftragte gegenüber dem Auftraggeber für getreue und sorgfältige Ausführung des ihm übertragenen Geschäfts. Das Auftragsrecht verweist damit bei berufstypischen Leistungen auf ein Vorgehen nach dem Standard des jeweiligen Berufes. Der Arzt schuldet eine auf die Wiederherstellung der Gesundheit des Patienten gerichtete Behandlung nach den Regeln der ärztlichen Kunst. Das bedeutet, der Patient hat zu beweisen, dass der Arzt oder die Klinik den Vertrag verletzt hat, er dadurch einen Schaden erlitten hat und zwischen Schaden und Vertragsverletzung ein adäquater Kausalzusammenhang besteht. Ein Verschulden muss nicht bewiesen werden, da dieses vermutet wird. Eine vertragliche Beschränkung der Arzthaftung auf grobe 
Verstösse gegen die Sorgfaltspflicht ist nicht möglich.

Zugleich haftet der Arzt unabhängig von einem Vertrag (ausservertraglich) gegenüber seinem Patienten aus deliktischer Handlung.

Es stellt sich nun die Frage, ob sich an den bisherigen haftungsrechtlichen Grundsätzen etwas ändert, wenn der Arzt als Arbeitnehmer einer bzw. seiner Aktiengesellschaft tätig wird.

Grundsätzlich kommt in diesem Fall der Behandlungsvertrag nicht zwischen behandelndem Arzt und Patienten zustande, sondern zwischen der Aktiengesellschaft und dem Patienten. Das bedeutet, dass nach vertraglichen Regeln nur die Aktiengesellschaft ins Recht gefasst werden kann und der Arzt daneben nur aus Deliktsrecht haftet. Aufgrund der Verschuldensvermutung, die eine wesentliche Erleichterung für den Geschädigten in der Geltendmachung seines Anspruchs darstellt, machen in den allermeisten Fällen die Geschädigten jedoch vertragliche Schadensersatzansprüche geltend. Die Verschiebung der vertraglichen Haftung vom Arzt auf seine Aktiengesellschaft ergibt sich auch für alle weiteren Vertragsverhältnisse, die ein Praxisbetrieb mit sich bringt. Zu denken ist an Verträge mit Personal, Lieferanten, Versicherungsgesellschaften und Banken. Zu beachten ist allerdings, dass, wenn die Ärzte neben ihrem Anstellungsverhältnis zugleich Verwaltungsrat der Aktiengesellschaft sind, eine zusätzliche Haftung der Aktiengesellschaft für ihre Organe, aber auch eine persönliche Haftung der Organe, besteht.

\section{Steuerrechtliche Aspekte}

Die Gründung einer AG oder $\mathrm{GmbH}$ mittels Sacheinlage der Aktiven und Passiven der bisherigen Arztpraxis löst keine Steuerfolgen aus. Allenfalls vorhandene stille Reserven können einkommenssteuerfrei auf die juristische Person übertragen werden.

Beim Arzt, der neu als Angestellter seiner neugegründeten Aktiengesellschaft bzw. GmbH tätig wird, kommt es zu einem Wechsel von einer selbständigen zu einer unselbständigen Erwerbstätigkeit. Dadurch lassen sich unter Umständen erhebliche Steuereinsparungen erzielen.

Zwar wird der mit dem Betrieb der Praxis erzielte Gewinn auf der Stufe der juristischen Person mit der Ertragssteuer erfasst, der Arzt ist allerdings nur noch für das bezogene Salär, die ausgeschütteten Dividenden und sonstige Vergütungen der Gesellschaft (z.B. Darlehenszinsen) einkommenssteuerpflichtig und nicht mehr für den gesamten mit dem Betrieb der Pra- xis erzielten Gewinn. Eine doppelte steuerliche Erfassung der erzielten Praxisgewinne auf Stufe Gesellschaft und anschliessend auf Stufe Aktionär lässt sich im Umfang des festgelegten Salärs und durch die Verzinsung von Aktionärsdarlehen vermeiden. Allenfalls kann es steuerlich vorteilhaft sein, einen Teil der Gewinne in der Aktiengesellschaft zu thesaurieren, um so Einkommenssteuern beim Arzt zu vermeiden. So können im übrigen Sozialversicherungsbeiträge vermieden werden, welche im Bereich der ersten Säule (AHV, ALV usw.) ohnehin meistens nicht mehr rentenbildend sind und daher eine reine Steuer darstellen.

Bedeutende Steuervorteile ergeben sich im weiteren aus der Verschiebung des Steuerdomizils, welche vielfach mit der Umwandlung in eine Aktiengesellschaft verbunden ist. Im Gegensatz zum selbständig tätigen Arzt wird beim unselbständigen Arzt das Erwerbseinkommen nicht mehr am Geschäftsort, d.h. am Standort der Praxis, sondern an dessen Wohnort besteuert. Damit kann ein Arzt seine Einkommenssteuerbelastung mit der Umwandlung seiner Arztpraxis in eine AG massiv senken, wenn sein Wohnort gegenüber dem Standort der Praxis eine tiefere Steuerbelastung aufweist. Der Standort der Arztpraxis in einer sogenannten Steuerhölle führt damit nicht unweigerlich $\mathrm{zu}$ einer hohen Einkommenssteuerbelastung beim Arzt, wenn er seinen Wohnort steuergünstig wählt.

Die grössten steuerlichen Vorteile beim Betrieb einer Arztpraxis im Rahmen einer AG ergeben sich bei der Übertragung der Praxis an Dritte oder an Nachkommen. Unter Einhaltung gewisser Voraussetzungen fallen auf den dabei erzielten Gewinnen keinerlei Einkommenssteuerfolgen an. Überdies ist in Fällen, bei denen mehrere Ärzte zusammen eine Gruppenpraxis führen, eine Übertragung von Anteilen der Gesellschaft sehr einfach möglich und bleibt für den Übertragenden ebenfalls meistens ohne Einkommenssteuerfolgen. Dagegen führen bei einer Personengesellschaft dieselben Transaktionen zu hohen Einkommenssteuerfolgen.

Nicht zu unterschätzen sind die Vorteile für Gruppenpraxen im Bereich der Mehrwertsteuer. Die unsägliche Praxis der Eidgenössischen Steuerverwaltung, gegebenenfalls bei der Weiterfakturierung von Infrastrukturdienstleistungen an Mitglieder der Gruppenpraxis die Mehrwertsteuer von $7,6 \%$ zu überwälzen und die dann beim belasteten Arzt mangels Vorsteuerabzugsmöglichkeit als Schattensteuer (auf Personalund Raumkosten) hängenbleibt, findet bei der AG keine Anwendung. Diese unnötige Mehr- 
wertsteuerbelastung lässt sich mit der Umwandlung der Gruppenpraxis in eine AG vollständig eliminieren.

\section{Fazit}

Die geltenden gesetzlichen Grundlagen ermöglichen es in vielen Kantonen, eine Arztpraxis in der Rechtsform einer juristischen Person zu führen. Es ist zu hoffen, dass Kantone, in denen diesem Ansinnen noch kantonale Regelungen entgegenstehen, ihre Gesetzgebung nächstens anpassen.

Soweit eine Arztpraxis in der Rechtsform einer juristischen Person geführt wird, gilt neu diese $(\mathrm{AG} / \mathrm{GmbH})$ als Erbringerin der ambulant erbrachten medizinischen Dienstleistungen. Sie kann diese mittels einer eigenen KVG-Nummer mit den jeweiligen Kostenträgern abrechnen, was bei einer Gruppenpraxis zu einer administrativen Vereinfachung führt.

Zivilrechtlich ist die Umwandlung einer in Form einer Personengesellschaft (Einzelfirma/ Kollektivgesellschaft) geführten Arztpraxis mit geringem Aufwand durchzuführen. Sämtliche Vertragsbeziehungen entstehen nach der Um- wandlung ausschliesslich mit der juristischen Person, auch die Patientenverträge. Dies führt zu einer Beschränkung der vertraglichen Haftung auf die juristische Person, womit das Privatvermögen des Arztes für diese Ansprüche nicht haftet und geschützt werden kann.

Nicht nur aus haftungsrechtlicher Sicht, sondern insbesondere auch aus steuerlicher Sicht kann sich die Umwandlung einer Arztpraxis in eine juristische Person in sehr vielen Fällen lohnen. Die grössten Steuervorteile ergeben sich vor allem bei anstehenden Nachfolgeregelungen sowie beim Verkauf der Praxis. Aber auch bei der laufenden Besteuerung lassen sich je nach Konstellation massgebliche Steuervorteile realisieren. Bei Gruppenpraxen können bei der AG die Wechsel der beteiligten Ärzte wesentlich steueroptimaler als bei Personengesellschaften durchgeführt werden. Schliesslich lassen sich im Bereich der Mehrwertsteuer unnötige Belastungen vermeiden.

Eine sachverständige Überprüfung der Frage, welche Vorteile sich aus der Umwandlung der eigenen Arztpraxis bzw. der bestehenden Gruppenpraxis in eine juristische Person ergeben, ist daher unseres Erachtens in jedem Fall angezeigt. 